\title{
Fuzzy Interest Forwarding
}

\author{
Kevin Chan \\ US Army Research Laboratory \\ kevin.s.chan.civ@mail.mil
}

\author{
Bongjun Ko \\ IBM T. J. Watson Research Center \\ bko@us.ibm.com
}

Spyridon Mastorakis
UCLA
mastorakis@cs.ucla.edu

\author{
Lixia Zhang \\ UCLA
}

lixia@cs.ucla.edu

\begin{abstract}
In the current Named Data Networking implementation, forwarding a data request requires finding an exact match between the prefix of the name carried in the request and a forwarding table entry. However, consumers may not always know the exact naming, or an exact prefix, of their desired data. The current approach to this problem-establishing naming conventions and performing name lookup-can be infeasible in highly ad hoc, heterogeneous, and dynamic environments: the same data can be named using different terms or even languages, naming conventions may be minimal if they exist at all, and name lookups can be costly. In this paper, we present a fuzzy Interest forwarding approach that exploits semantic similarities between the names carried in Interest packets and the names of potentially matching data in CS and entries in FIB. We describe the fuzzy Interest forwarding approach and outline the semantic understanding function that determines the name matching.
\end{abstract}

\section{CCS CONCEPTS}

- Networks $\rightarrow$ Data path algorithms; Network protocol design;

\section{KEYWORDS}

Named Data Networking, fuzzy matching, Interest forwarding

\section{ACM Reference Format:}

Kevin Chan, Bongjun Ko, Spyridon Mastorakis, Alexander Afanasyev, and Lixia Zhang. 2017. Fuzzy Interest Forwarding. In AINTEC '17: Asian Internet Engineering Conference, November 20-22, 2017, Bangkok, Thailand. ACM, New York, NY, USA, 7 pages. https://doi.org/10.1145/3154970.3154975

\section{INTRODUCTION}

Named Data Networking (NDN) [12, 26, 27] is a new networking approach that realizes the Information-Centric networking (ICN) vision by focusing on data retrieval by names rather than on pointto-point data delivery based on addresses. The foundation of NDN is the shared use of hierarchical and semantically meaningful namespace between application and network layers, enabling applications to request data by names using Interest packets. NDN routers use names carried in Interests to decide where to forward each Interest if the requested data is not available in the local content store

ACM acknowledges that this contribution was authored or co-authored by an employee contractor, or affiliate of the United States government. As such, the United States government retains a nonexclusive, royalty-free right to publish or reproduce this article, or to allow others to do so, for government purposes only.

AINTEC '17, November 20-22, 2017, Bangkok, Thailand

(C) 2017 Association for Computing Machinery.

ACM ISBN 978-1-4503-5551-3/17/11 ..\$15.00

https://doi.org/10.1145/3154970.3154975
(CS), creating pending state for the forwarded Interest. When the data is found, routers use these pending states to forward data back to one or more requesters. Both Interest forwarding and data returning use a prefix match operation: an Interest name finding the longest prefix from the forwarding information base (FIB); a data name finding prefix match(es) from the pending Interest tables (PIT). Note that applications need to know at least a prefix of the data (e.g., "/park/yellow-stone/lost\&found/dog/...") in order to retrieve it. In cases where consumers and producers do not have a full agreement on the exact terminology (e.g., when instead of "dog", consumers or producers use "puppy", "canine", or "hound"), consumers may have problems retrieving data, even though these names are semantically close and the retrieved information can be useful in finding the lost pet.

In this work, we propose a fuzzy Interest forwarding augmentation to NDN routers that enables discovery and retrieval of data using approximate knowledge of the data namespace. In particular, as we explain in Section 3, such fuzzy forwarding can be beneficial in highly dynamic and heterogeneous environments, e.g., ad hoc encounters of tourists in national parks, where the exact namespace knowledge among communicating parties is either infeasible to obtain or costly to discover. Semantic-aware fuzzy forwarding can enable NDN routers to forward Interest packets with controlled uncertainty and receive potentially relevant and useful data most of the time. The returned data can also provide a feedback loop that allows applications to learn about the namespace of available data.

The contribution of this paper is three-fold:

- Identifying new challenges of name discovery in highly heterogeneous networked environments;

- Introducing the concept of fuzzy interest forwarding; and

- Proposing an initial approach to the solution development.

We provide an overview of the work related to our proposed approach in the following section and a motivating use case in Section 3. Section 4 describes the overview of fuzzy Interest forwarding, and then we present the details of the semantic name matching and look-up in NDN contexts in Section 5. Finally, Section 6 discusses some preliminary evaluation results and additional challenges in realizing the proposed approach.

\section{RELATED WORK}

This work proposes an extension to the Named Data Networking forwarding mechanism that leverages results from semantic understanding research from the past and recent work. Below we briefly review related concepts and work related to semantic understanding. 
Fuzzy Information Retrieval. With the emergence of the Semantic Web, there have been efforts to do fuzzy information retrieval based on the data and ontologies $[4,7,8]$ in the context of web searches. Given a keyword, Internet search engines comb through the database that has a complete knowledge of the world to look for every potential match. The search results can then be further confined/reordered based on pre-existing information about the user (e.g., user's location, pre-configured preferences, previous search history, etc.). In some approaches, there may be a lack of consistency across similar data across multiple ontologies. While there is a significant amount of work in the area of web search, there is a clear benefit to studying fuzzy information forwarding, as the information matching and networked information flows (both upstream and downstream) are integrated.

Attribute-Based Scheme. There exists a number of proposals for attribute-based or name-value pair resource discovery schemes. Adjie-Winoto et al. [1] proposed the Intentional Naming System (INS) and approach that routes information using predefined naming conventions. The INS resolvers are responsible for information routing as well as updating any additional named data in the network. To address uncertainty in the name-value pairs, INS allows for wildcard values, but do not exploit any similarities between the resources or their names. INS assumes a static environment with a given set of attribute names, thus it does not admit any dynamic learning or feedback of the data or namespace. It is designed as an application overlay, running on top of an existing (IP) network, instead of providing network layer delivery services.

Naming Conventions for Name Discovery. In NDN, when users or applications know the exact data names, they simply express Interests for this data. However, for dynamically produced data or data that can be produced by different publishers (i.e., distributed data production), the exact name may not be known a priori. NDN allows consumers to fetch data using prefixes, which can bring back any data under that prefix. Based on the received data and following established naming conventions, the user can deduce what (exact) name(s) to use to fetch the next (other) data piece(s). For example, to discover current alerts about Yellowstone park, an application can send an Interest for "/park/yellowstone/alerts", which returns a version of the alerts page, which could include additional information about the version, e.g., "/park/yellowstone/alerts /_v=2017-04-23”. With understanding of versioning naming conventions, the application can then request a more recent version or one from several days ago. Establishing naming conventions can be a powerful tool in name discovery; however it assumes all communicating parties share well established naming conventions a priori.

Metadata for Name Discovery. The naming conventions provide knowledge about the namespace structure. In more dynamic environments or where it is impossible to define a standard set of namespace categories, one may consider to use "metadata" to describe names that can appear under a given prefix. For example, a published version of metadata for the park, (e.g., "/park/yellowstone /lost\&found/_metadata”) can list all report categories for the lost and found items and pets, currently known to the ranger station. Using metadata for name discovery costs additional round trips, can be expensive when the list of categories is large, and have a limited value when categories could not be aggregated at a central point (e.g., when park visitors do not have connectivity to the ranger station).

\section{MOTIVATION \& USE CASE}

Our target scenarios are network environments with high degrees of heterogeneity and dynamics, where the namespace of the communicated data can be defined only partially. As a specific use case in this paper, we use potential NDN-based ad hoc communication between visitors in popular areas of a national park (e.g., searching and reporting missing pets near Old Faithful geyser in Yellowstone national park); we believe similar scenarios can be found in many other applications with high uncertainty and dynamics.

Communication between park visitors are typified by significant dynamics, limited knowledge, and lack of resources. Specifically, users and devices' mobility lead to network topologies and link states vary with time. Visitors come from different parts of the world, having different understanding of terminology to describe data, contributing to heterogeneity of data naming and naming convention. There may also be limitations in resources and limited (or no) access to the infrastructure-assisted information retrieval. For example, reports about lost pets or sighting of wild animals may not be able reach a ranger station for information aggregation, but would be immediately useful to visitors in the area.

In this paper, we consider non-malicious users trying to publish animal sighting reports and users who want to retrieve information, based on some partial knowledge of the reports' name. We assume that there is a large report amount available in the network and limited communication budget, making infeasible to retrieve the entire dataset or even enumerate the namespace. We assume that users (applications running on their devices) have some namespace understanding, e.g., they would know that all lost\&found reports in the park are published under the "/park/yellow-stone /lost\&f ound" prefix and all wild animals sightings near Old Faithful under "/park/yellowstone/old-faithful/alerts". To retrieve data, users construct Interests under the specific prefixes, adding potentially ambiguous parts to describe the desired information ("dog"/"pup" when searching for a lost pet, "bison"/"buffalo" or "bear"/"grizzly" when looking for wild animal alerts). The constructed Interests may also include suffixes to describe the requested information type, such as "info" or "video" for a text description or a video feed, and other meta parts (e.g., version and segment numbers). Users then express such Interests to the NDN network that includes their ad hoc peers or any ranger stations in sight, which utilizes fuzzy interest forwarding and data matching to retrieve data semantically close to the request, but not necessarily has the same exact prefix. After receiving initial reply, users can refine their question and retrieve other associated data, e.g., other segments of video report.

\section{FUZZY INTEREST FORWARDING (FIF)}

This section describes the architecture of augmenting NDN with Fuzzy Interest Forwarding (FIF). As highlighted in Figure 1 with grayed "FF" blocks, we propose to add fuzzy matching operations at two stages as an Interest packet goes through an NDN forwarder: 


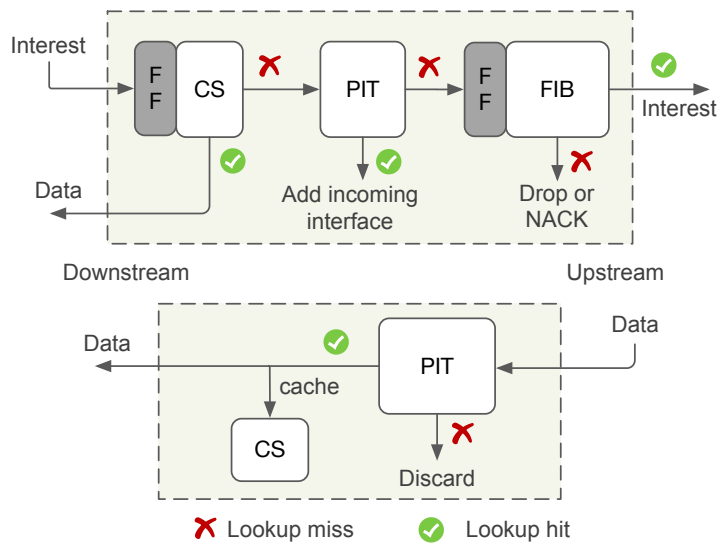

Figure 1: NDN forwarding strategies with fuzzy forwarding

the Content Store (CS) and the Forwarding interest base (FIB). To match the retrieved Data with the Interest in the PIT, we plan to rely on a recently proposed interest digest mechanism, highlighted later in this section.

\subsection{Augmented Interest Forwarding}

Overall, the fuzzy Interest forwarding mechanism shown in Figure 1 is similar to the standard Interest processing by an NDN router [2, 25, 27]: returning data if there is a CS match, creating a new PIT entry or aggregating with an existing one, forwarding the Interest based on a matched FIB entry. ${ }^{1}$ However, matching in the $\mathrm{CS}$ and FIB is performed not just based on the prefix match, but also considers the semantic closeness between some of the name components. In other words, when looking up the CS and FIB, an NDN router uses a part of the Interest name as usual to select a set of potential candidates, then uses one or more name components to calculate fuzzy semantic distance with the candidates, potentially following with further matching on the name suffix (exact or fuzzy). We leave to the future research the decision of how exactly the router determines which part of the name should be matched exactly and which fuzzy. One potential direction is to designate a dedicated name component type.

When an Interest fails to find a fuzzy match in CS (i.e., there are no data matching the non-fuzzy prefix or the fuzzy match resulted in a low-confidence match, see Section 5), it is fuzzy matched with entries in the FIB. For such fuzzy-forwarded Interests, the returned Data most likely will not share the same prefix. One way to address this issue is to use a recently proposed interest digest mechanism [21] to uniquely identify the Interest from the PIT that fetched the returned Data packet. Whenever a node $N_{1}$ finds a (fuzzy) matching data for an Interest it received from a neighbor node $N_{2}, N_{1}$ returns the data to $N_{2}$ together with the digest (i.e., a cryptographic hash) of the Interest, so that $N_{2}$ can uniquely identify the Interest in its PIT that fetched the returned data packet. This way, the Data forwarding path does not require any additional changes to support FIF.

\footnotetext{
${ }^{1}$ The actual forwarding decision is made by the forwarding strategy, which we omit for simplicity.
}

FIF also does not require changes to the match between Interests in PIT: Interests carrying different names will create separate entries in PIT, regardless of their semantic distance. First, it maintains the flow balance because each Interest retrieves at most one (potentially same) data packet. Second, keeping the original PIT matching logic prevents fuzzy matching of already fuzzy matched names, which may result in a semantic similarity dilution of results returned to the original Interest.

\subsection{Design Considerations}

4.2.1 Semantic-Based Cache Replacement. As nodes are generally storage-limited, the CS generally apply a content replacement policy, such as least recently used (LRU), first-in-first-out (FIFO), or others. CS can also utilize fuzzy matching in the replacement strategy, determining which data should be stored based on a given semantic similarity metric. For example, CS may prioritize removal of data packets semantically close to each other, keeping only one of them. This way, CS can conserve its limited storage space, yet be able to return (hopefully useful) data to different incoming Interests.

4.2.2 Geo-Based FIB. To perform a meaningful fuzzy match in FIB, it needs to be filled with entries related to data. We assume that producers or repositories announce prefixes, which are propagated through the network via routing protocols, such as NLSR [10, 15]. In the ad hoc context, where usually there is a limited number of interfaces per node, it is possible to use a geo-based FIB (gFIB) [9]. gFIB is still populated through announcements (proactive) or data discovery interests (reactive) from data carriers (producers, publishers, or data mules). However, instead of designating a "next-hop" interface, gFIB entries are associated with geographical coordinates or directions (angles). Depending on from which direction an Interest has been received, a gFIB entry may tell to forward (re-broadcast) or drop the Interest.

4.2.3 Use of Fuzzy Match. Depending on the network and information context, the fuzzy matching described in Section 5 can be applied in different ways. The most appropriate approach is an open question and we leave it for our future work. In the CS context, fuzzy matching needs to find at most one entry for each Interest, as only one Data can be returned. Depending on the similarity threshold-another open question and an important design choice-a match can either always return a result, provided there is data that matches the non-fuzzy Interest part, or return when a "reasonably" close match is found.

In the FIB context, the fuzzy match can return zero or multiple entries, found with or without defined semantic similarity bounds. These entries can be used either (1) to forward the Interest based on the next-hop information from "all" entries, (2) based on top- $k$ FIB entries, or (3) to forward based on the "best" entry in terms of the distance measure. In cases when an Interest fails to find any match in FIB, the Interest can be either dropped, or forwarded to a default place where more advanced semantic matching can be performed.

4.2.4 Forward or Wait Challenge. The incoming Interest can be semantically matched with different (set of) entries, depending on the current CS and FIB state that changes with each new data or routing announcement. This poses a challenge for the router 


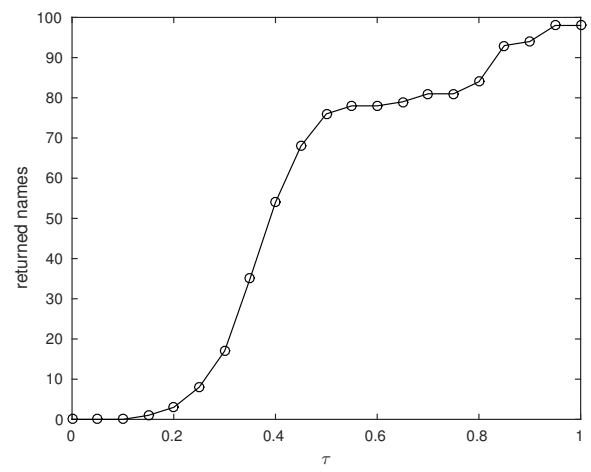

Figure 2: Names vs $\tau$ using WUP over the Wordnet Synset corpora matching wildebeast to the UCI Zoo dataset.

whether for each received Interest to immediately apply the fuzzy logic, or delay it by some time in the hope that it will receive more relevant data (requested by previously sent Interests) or prefix announcements. The more router waits, the more "precise" fuzzy decision it can make, while diminishing the requester satisfaction. Specific to the fuzzy name matching, we can also envision user feedback to refine the returned fuzzy matched data (further discussion in Section 6).

\section{FUZZY NAME MATCHING AND LOOKUP}

In this section, we discuss in detail the issues of matching the names ${ }^{2}$ in NDN by their semantic similarity and how to perform the look-up of the semantically matching names in FIB and CS.

\subsection{Fuzzy Name Matching}

With fuzzy name matching, a name is matched to other name(s) in CS and FIB based on a certain measure of their semantic similarity. ${ }^{3}$ To formally define such a similarity measure, let us denote by $D\left(n, n^{\prime}\right)$ a distance function between two names $n$ and $n^{\prime}$, where a small $D\left(n, n^{\prime}\right)$ means $n$ and $n^{\prime}$ are similar (0 for the same or "equivalent" names, close to \pm 1 for very different names). Then the fuzzy matching of a given name $n$ (of an Interest) to a set of names $S$ (in $\mathrm{CS}$ or FIB) can be defined as either

- finding a name $s$ in $S$ that minimizes $D(n, s)$ (Best-Match), or

- finding names in $S$ such that $D(n, s)<\tau$ for some threshold constant $\tau>0$ (Qualified-Matches).

In practice, we expect the combination of the above matching methods to be most useful, which returns the best-matching name only if its distance to the given name is within a certain threshold. In what follows, we review two semantic distance measure categories and methods to obtain them in NDN. We first consider case when only one name component with a single word is matched against another single word. We discuss more complex fuzzy matching cases on multiple name components in Section 5.4.

\footnotetext{
${ }^{2}$ For simplicity, we talk as if fuzzy match is performed on the whole name. As we described in Section 4 , the actual fuzzy match may include only certain components, either designated by the consumer or producer.

${ }^{3}$ In this paper we do not consider the syntactic similarity, which can be efficiently handled by existing methods [20].
}

5.1.1 Ontological matching. With ontological matching, the similarity between two names is found based on a pre-defined name ontology given by a lexical database such as WordNet [19]. A variety of the semantic distance measures have been proposed and used in the literature, but essentially they are typically defined by some function of the topological distance between the nodes in the ontological tree.

In Figure 2, we present the result of an experiment to demonstrate how we envision FIF would be affected by the choice of the threshold parameter $\tau$ in the ontological distance measure. We use an example from the UCI Machine Learning Repository Zoo Data Set [16] as the input data, the Synset in WordNet corpus as our ontology, and the WUP Similarity score by Wu and Palmer [24] as the semantic similarity function. The figure shows an (obvious) increase in the number of similar names being returned as the threshold $\tau$ increases. We also see a sharp transition at around $\tau=0.3$, which indicates the parameter choice plays an important role as a control knob to tradeoff the accuracy with the overhead of fuzzy forwarding.

Since the ontology-based matching uses a pre-defined dictionary curated by people over time, it can provide a well-defined set of semantically similar words for a word majority used by humans. The major drawback is that a large database needs to be loaded in NDN routers (consider NDN forwarder running on users' phones in our example) and queried each time a name is to be matched against others, which would prohibitively increase the cost both in terms of memory and processing time. For this reason, we envision this method would be appropriate when the fuzzy name matching can be performed in resource-rich computing nodes, e.g., at the ingress point of the network or server-scale middle boxes to which the Interest processing is delegated.

5.1.2 Contextual matching. At a high level, the contextual matching of words is done through the semantic distances learned statistically from a corpus of texts (documents, sentences, phrases) that they are used in. It operates under the assumption of so-called distributional semantic hypothesis, that is, words close in meaning tend to occur in the same piece of texts (same document or same sentence). While there are many different methods for learning the contexts, they generally work by learning a mapping function that converts the words into fixed-length vectors in some latent vector space of much smaller dimension than the original corpus of words. Then the distance between the mapped vectors represents the semantic distance between the words (See Figure 3).

There are two broad classes of mapping functions and how they are learned: context-counting methods and context-predicting ones. The former (more classical) approaches, represented by Latent Semantic Analysis (LSA) [13], discover the latent features of the words based on their co-occurrence counts in the set of documents. Whe latter (more recent) breed focuses on the "prediction context" that indicates a word's influence on predicting other surrounding words in the text [14]. Details on the difference and similarity between these two approaches can be found in literature [5].

With these approaches, our distance function $D\left(n, n^{\prime}\right)$ would be defined as $D\left(n, n^{\prime}\right)=d\left(v, v^{\prime}\right)$, where $v=F(n)$ and $v^{\prime}=F\left(n^{\prime}\right)$ are the respective n-dimensional vector representations of names $n$ and $n^{\prime}$ determined by the name-to-vector mapping function $F$, and $d(\cdot)$ is 


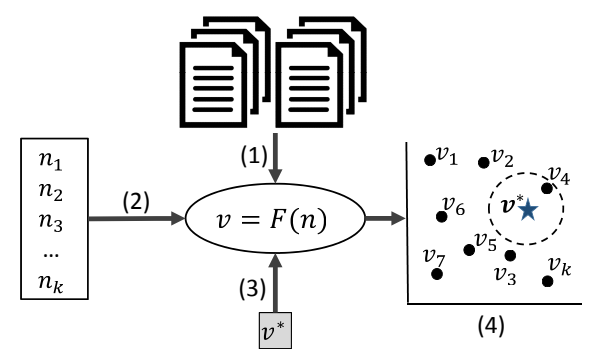

Figure 3: Constructing and using vector space model for contextual similarity distance between the words. (1) A nameto-vector mapping function is learned from a corpus of texts, (2) names in CS and FIB are mapped to vectors, (3) a new name (query) is mapped to a vector, and (4) semantically similar names are found in the vector space through some distance measure between the vectors.

a distance function in the vector space. A typical distance measure is the cosine distance:

$$
d(v, w)=1-\frac{v \cdot w}{\|v\|\|w\|},
$$

where $v \cdot w$ is a dot product of two vectors $v$ and $w$, and $\|v\|$ and $\|w\|$ their magnitudes.

These approaches have distinct advantages over the ontologybased approaches. First, the semantic relationship between the names is represented by a compact vector model, hence not requiring the entire database of the names and their relationship to be loaded in memory for fuzzy matching. Also, these methods can inherently provide the semantically similar names within the context of how the names of data are being requested. Finally, the distance calculation between the words are done through simple algebraic operation in vector space, making them adequate for resourceconstrained NDN routers. Due to these benefits, in what follows we further explore some details of how the contextual matching can be effectively utilized in NDN environments.

\subsection{Constructing Vector Space Model of Names}

A potential issue of using the vector space model for contextual name matching is that its applicability and effectiveness depends on the dataset used to train and build the model since a similarity between two names can be found only when they have been seen in the training dataset. Here we present a few alternatives and their pros and cons when used in NDN contexts.

One approach is to build the vector model with the NDN names observed (published and used). The name contexts can be obtained from the hierarchical name constructs (prefixes and suffixes) as the "sentences" in which the individual words are used. The challenge, however, is that names only can provide the context to only a limited extent so the accuracy of the model may be low. Also, because the model is built and evolved dynamically as new observations are made, it needs to be somehow synchronized dynamically across all routers. ${ }^{4}$

\footnotetext{
${ }^{4}$ It should be possible to have each NDN router build its own model based on what it observes, though it implies additional challenges regarding the model being more sparse and the potential network-wide instability of the fuzzy forwarding due to heterogeneous forwarding decisions.
}

Another approach at the other end of spectrum is to build a model from a large corpus of public data set, such as Wikipedia documents, that includes a rather comprehensive set of words and their contexts. With this approach, a pre-built, static model with relatively good coverage can be used across all NDN routers. The downside, however, is that the vector space model itself can become very large, and there is still a chance that some names may not be present in the model.

A hybrid of the above two approaches can potentially strike a good balance, which uses corpora of online documents and other meta information on the data themselves that exist in the network in addition to the associated names, in order to enrich the contextualization of the vector space model. How to synchronize the learned model across the network still remains an open problem, to which a variety of solution strategies can be considered-from a centralized control plane, to an NDN-approach to model distribution (by assigning the models unique names), to a decentralized (possibly localized) model synchronization between nearby nodes.

\subsection{Fuzzy Name Look-up in Vector Space}

The final step of fuzzy forwarding is to find, among all names in FIB and CS, the nearby ones to a given name in the vector space (i.e., step (4) in Figure 3). Whether the goal is to find the most similar one or those within a certain distance threshold, this step involves searching for nearest neighbor(s) in the vector space. A naïve method for the nearest neighbor search would evaluate the pair-wise distances between the query vector and all vectors, resulting in $O(n)$ search cost, which could take prohibitively long in resource-constrained routers. To address that, we suggest the use of approximate nearest neighbor (ANN) search methods such as Locality Sensitive Hashing (LSH) [23], which provide $O(1)$ or $O(\log n)$ look up time at the small expense of additional memory requirements for indexing data points.

As a proof of concept, we conduct a small experiment to measure the performance of an ANN search using LSH Forest [6] method for 400-dimensional vector space. In Figure 4(a), we compare the ANN query response times of LSH Forest method with different parameters against a brute-force linear search as we vary in the $x$ axis the total number of data points to which the given query vector is compared against. Figure 4(b) shows the average accuracy of the ANN search result returned by LSH Forest method. The results indicate that LSH Forest method (or other ANN methods) produce reasonably accurate $\mathrm{NN}$ search results with almost constant lookup time, making them viable choices for fuzzy name search in resource-constrained NDN routers.

In addition, it would be beneficial to quickly determine whether a name semantically matched to the given one exists in a set of names (e.g., CS or FIB for individual outgoing interface) so that the actual look-up is performed only when there is a match. For such a membership query, the use of Bloom filter has been proposed in literature for exact name query in NDN [22]. In the similar spirit, we could explore the use of locality-sensitive Bloom filters (LSBF) [11] for the approximate membership query in the vector space model of the names. 


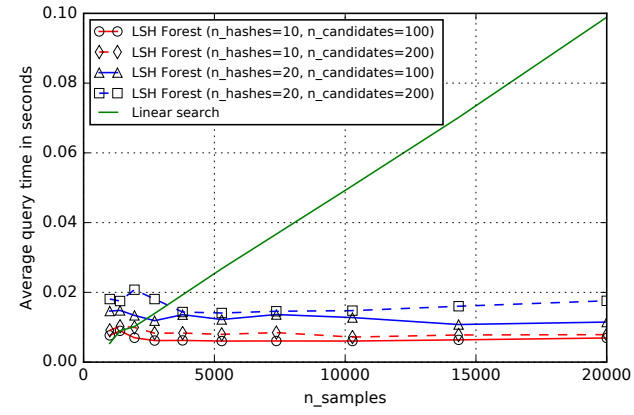

(a) Comparison of query response time

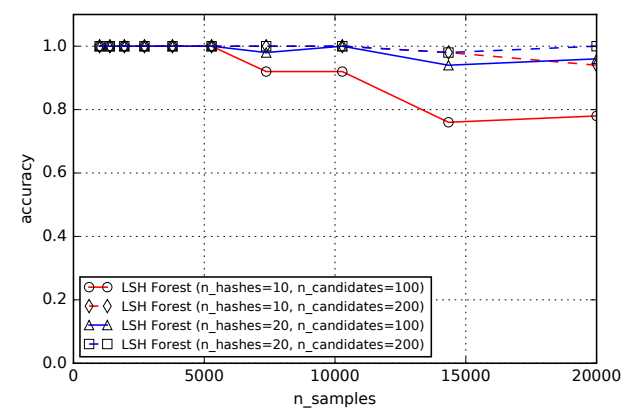

(b) Accuracy of LSH Forest method for nearest neighbor search

Figure 4: Performance of ANN search using LSH Forest algorithm.

\subsection{Multi-Component Fuzzy Match}

NDN names are shared between the application and the network, therefore, as we highlighed in Section 4, some parts of the name still need to be exactly matched. For example, "/park/yellowstone /lost\&found" should always be matched exactly (i.e., non-fuzzy) to avoid retrieval of irrelevant data from other parks or wild animal alerts instead of the lost\&found reports. However, how many components of the name can be fuzzy-matched (who and how they are designated) is an open question. Here we discuss two approaches to realize fuzzy matching that considers multiple components of an NDN name, having their own advantages and limitations that we plan to explore in our future research.

One approach to realize the multi-component fuzzy match is to make it a superposition of the word-by-word (component-bycomponent) fuzzy matches at the corresponding positions in the sequences. Specifically, given two multi-component sequences, the measure of their semantic distance could be some combination of the component-wise distances at individual positions. However, the name hierarchy itself can be different from one namespace to another (e.g., ". ./animal/dog/.." versus ". ./animal/mammal /canine/hound/..."). Therefore, it may not be sufficient to compare the words at the same level only, and the distance measure may need to consider all cross-level distances, possibly assigning higher weights to the same-level comparisons to account for a higher relevance in the overall assessment of the similarity.

Another approach is to compare multi-component sequences as if they are flat ones. In other words, one can build a vector space model, not for individual words, but for the entire sequences by treating them as phrases or sentences-in much the same way used for topic modeling and sentiment analysis of texts. One then would compare the entire "sentences" in the vector space to find their similarity; e.g., the "sentence" of ". ./pet/puppy/hound/.." would be mapped to a vector close to one mapped from ". . /animal /canine/dog/..”.

\section{PRELIMINARY EVALUATION \& IDENTIFIED CHALLENGES}

\subsection{Preliminary Evaluation Results}

We implemented FIF $^{5}$ in ndnSIM [17] using Google's word2vec tool [18] for fuzzy matching. For a quick proof of concept, we performed single name component fuzzy matching in a simple topology of three nodes; a consumer expressing 10 interests per second, a producer responding to the interests, and an intermediate router. We run the simulation 5 times, each run lasts 60 seconds (thus the consumer expresses 600 prefixes total).

We compare the data retrieval performance of FIF to that of Exact Match Forwarding (EMF). Figure 5(a) shows that the FIF retrieval rate is $\approx 4$ times higher than EMF for small FIB sizes, and $\approx 5$ times better for larger FIB sizes. Figure 5 (b) shows that FIF has slightly better performance than EMF when the semantic similarity threshold is high, while the retrieval rate increases as we reduce the similarity threshold.

At the same time, FIF introduces a relatively high demand on memory size. In our experiments, the word2vec tool required $98 \mathrm{MB}$ of additional memory per simulated node for a small-size model and 1.2 GB for a larger one (containing $71 \mathrm{~K}$ and $3 \mathrm{M}$ words respectively).

\subsection{Identified Challenges}

Resource Constraints. The current semantic similarity function, as described in Section 5 and highlighted in our preliminary evaluations in previous Section, can have a high demand on storage and computational resources (compared to exact matching-based forwarding), especially for nodes in resource-constrained environments. While the quantitative assessment of the approaches and their optimization call for further research efforts (in terms of memory requirements, processing speed, and possibly use of hardware acceleration), one way to get around this difficulty is to use default forwarding to direct interests that fail local matching to a more powerful node (i.e., semantic oracle) providing the semantic analysis for the fuzzy match computation. This removes the computation and storage cost on individual nodes, but still offers the FIF capability if adequate network bandwidth is available. In a bandwidth limited environment, we may consider either to use a fast, but possibly lower performing approach to locally carry out fuzzy matching, or to let non-fuzzy nodes perform forwarding according to the default routes.

Improving matching through feedback. When a forwarding node fails fuzzy matching in FIB lookup, another option can be sending a negative acknowledgment (NACK) to the requester, together with the most relevant names/name prefixes. This NACK can be potentially useful to the requester in two ways: a) to inform what contents may be available, and b) to guide the adjustment of the

\footnotetext{
${ }^{5}$ https://github.com/spirosmastorakis/FIF
} 


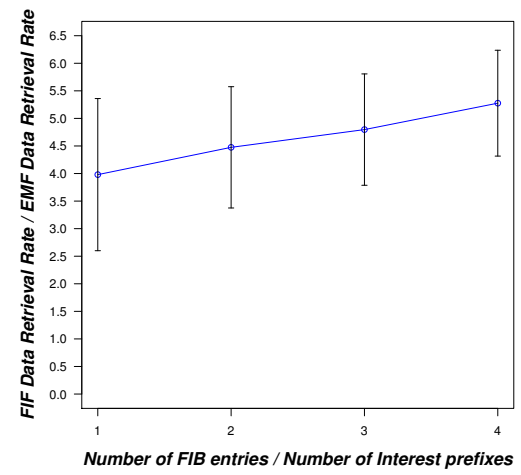

(a) Retrieval rate vs FIB size, 5 fuzzy matches, semantic similarity threshold $=0.4$

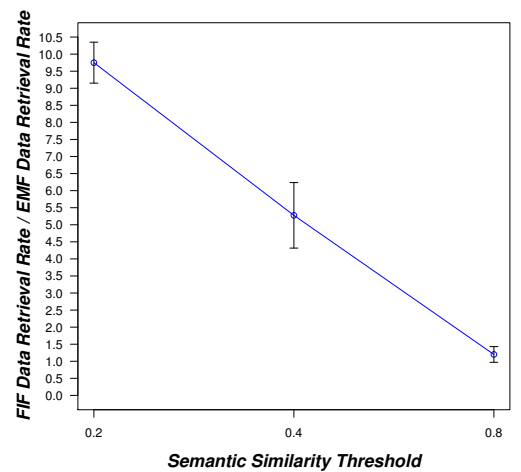

(b) Retrieval rate vs similarity threshold, FIB size $=4 \mathrm{x}$ prefixes, 5 fuzzy matches

\section{Figure 5: Data retrieval rate enhancement}

requested name and try again. A related challenge is how to incorporate this additional knowledge in the semantic model. One way could be to add dynamically-adjusted weights (or 'penalty') to the semantic distance of those NACK'ed, and another to create a companion model for "black lists". The details of how to do these systematically remain an open issue.

Attack Surface with Fuzzy Matching. Although one can stay assured that NDN's data-centric security design should be able to detect all false data, malicious parties may still try to abuse FIF to launch DoS attacks. Approaches similar to the one described in [3] can be explored as potential solutions.

\section{ACKNOWLEDGMENTS}

This work is partially sponsored by the U.S. Army Research Laboratory and the U.K. Ministry of Defence under Agreement Number W911NF-16-3-0001, and the National Science Foundation under awards CNS-1345318 and CNS-1629922. The views and conclusions contained in this document are those of the authors and should not be interpreted as representing the official policies, either expressed or implied, of the U.S. Army Research Laboratory, the U.S. Government, the U.K. Ministry of Defence or the U.K. Government.
The U.S. and U.K. Governments are authorized to reproduce and distribute reprints for Government purposes notwithstanding any copyright notation hereon.

\section{REFERENCES}

[1] William Adjie-Winoto, Elliot Schwartz, Hari Balakrishnan, and Jeremy Lilley. 1999. The design and implementation of an intentional naming system. ACM SIGOPS Operating Systems Review 33, 5 (1999), 186-201.

[2] Alexander Afanasyev et al. 2016. NFD Developer's Guide. Technical Report NDN-0021, Revision 7. NDN.

[3] Alexander Afanasyev, Priya Mahadevan, Ilya Moiseenko, Ersin Uzun, and Lixia Zhang. 2013. Interest Flooding Attack and Countermeasures in Named Data Networking. In Proc. of IFIP Networking 2013.

[4] Zeinab E. Attia et al. 2014. An Enhanced Multi-view Fuzzy Information Retrieval Model based on Linguistics. IERI Procedia 7 (2014), 90 - 95. https://doi.org/10. 1016/j.ieri.2014.08.015

[5] Marco Baroni, Georgiana Dinu, and GermÃąn Kruszewski. 2014. Don't count, predict! A systematic comparison of context-counting vs. context-predicting semantic vectors.. In ACL (1). 238-247.

[6] Mayank Bawa, Tyson Condie, and Prasanna Ganesan. 2005. LSH forest: selftuning indexes for similarity search. In Proceedings of the 14th international conference on World Wide Web. ACM, 651-660.

[7] Valerie Cross. 1994. Fuzzy information retrieval. fournal of Intelligent Information Systems 3, 1 (1994), 29-56.

[8] M. A. d. A. Leite and I. L. M. Ricarte. 2008. Fuzzy Information Retrieval Model Based on Multiple Related Ontologies. In Proc. of IEEE International Conference on Tools with Artificial Intelligence.

[9] Giulio Grassi, Davide Pesavento, Giovanni Pau, Lixia Zhang, and Serge Fdida. 2015. Navigo: Interest forwarding by geolocations in vehicular Named Data Networking. In IEEE 16th International Symposium on a World of Wireless, Mobile and Multimedia Networks (WoWMoM).

[10] AKM M. Hoque et al. 2013. NLSR: Named-data Link State Routing Protocol. In ACM SIGCOMM ICN Workshop.

[11] Yu Hua et al. 2012. Locality-sensitive bloom filter for approximate membership query. IEEE Trans. Comput. 61, 6 (2012), 817-830.

[12] V. Jacobson, D.K. Smetters, J.D. Thornton, M.F. Plass, N.H. Briggs, and R.L. Braynard. 2009. Networking Named Content. In Proceedings of the 5th International Conference on Emerging Networking Experiments and Technologies.

[13] Thomas K Landauer. 2006. Latent semantic analysis. Wiley Online Library.

[14] Quoc V Le and Tomas Mikolov. 2014. Distributed Representations of Sentences and Documents.. In ICML, Vol. 14. 1188-1196.

[15] Vince Lehman, A K M Mahmudul Hoque, Yingdi Yu, Lan Wang, Beichuan Zhang, and Lixia Zhang. 2016. A Secure Link State Routing Protocol for NDN. Technical Report NDN-0037. NDN Project.

[16] M. Lichman. 2013. UCI Machine Learning Repository. http://archive.ics.uci.edu/ $\mathrm{ml}$. (Last accesed on November 5 2013). http://archive.ics.uci.edu/ml

[17] Spyridon Mastorakis, Alexander Afanasyev, and Lixia Zhang. 2017. On the evolution of ndnSIM: An open-source simulator for NDN experimentation. ACM SIGCOMM Computer Communication Review 47, 3 (2017), 19-33.

[18] Tomas Mikolov et al. 2013. Google's word2vec tool. https://code.google.com archive/p/word2vec/. (2013)

[19] George A Miller. 1995. WordNet: a lexical database for English. Commun. ACM 38, 11 (1995), 39-41.

[20] Gonzalo Navarro. 2001. A guided tour to approximate string matching. ACM computing surveys (CSUR) 33, 1 (2001), 31-88.

[21] NDN Team. 2017. Interest Digest. https://redmine.named-data.net/issues/3333. (2017)

[22] Wei Quan, Changqiao Xu, Jianfeng Guan, Hongke Zhang, and Luigi Alfredo Grieco. 2014. Scalable name lookup with adaptive prefix bloom filter for named data networking. IEEE Communications Letters 18, 1 (2014), 102-105.

[23] Malcolm Slaney and Michael Casey. 2008. Locality-sensitive hashing for finding nearest neighbors [lecture notes]. IEEE Signal Processing Magazine 25, 2 (2008), $128-131$

[24] Zhibiao Wu and Martha Palmer. 1994. Verbs semantics and lexical selection. In Proceedings of the 32nd annual meeting on Association for Computational Linguistics. Association for Computational Linguistics, 133-138.

[25] Cheng Yi, Alexander Afanasyev, Ilya Moiseenko, Lan Wang, Beichuan Zhang, and Lixia Zhang. 2013. A Case for Stateful Forwarding Plane. Computer Communications 36, 7 (April 2013), 779-791.

[26] Lixia Zhang et al. 2010. Named Data Networking (NDN) Project. Technical Report NDN-0001. NDN Project.

[27] Lixia Zhang, Alexander Afanasyev, Jeffrey Burke, Van Jacobson, kc claffy, Patrick Crowley, Christos Papadopoulos, Lan Wang, and Beichuan Zhang. 2014. Named Data Networking. SIGCOMM Comput. Commun. Rev. 44, 3 (July 2014), 66-73. 